Perspectiva de Familia (Perspect. fam.) ISSN 2415-5187, año 2016, vol. 1, pp. 73-83

\title{
Familia y educación de la prudencia
}

Family and Education of Prudence

María Lorena Diez Canseco Briceño

Universidad Católica San Pablo, Perú

Correspondencia: Idiezcanseco@ucsp.edu.pe

Recibido: 09-12-2015

Aceptado: 02-03-2016

\section{Resumen}

El logro de la virtud es quizá una de las tareas más difíciles de llevar a cabo y sin embargo constituye la finalidad de la educación de la persona humana, aquello a lo que debe aspirar para poder alcanzar su perfeccionamiento. Este perfeccionamiento al que la persona humana está llamada, no lo puede alcanzar sólo valiéndose de sus propios medios, sino que requiere de la guía y conducción de otros que ya hayan recorrido el camino para el desarrollo de la virtud; la familia es el lugar por excelencia en donde el ser humano puede encontrar esta guía y conducción de la que hablamos, es ahí donde la vivencia y el desarrollo de las virtudes se puede dar de una manera natural, debido a que este es el ámbito en donde, por naturaleza, se da la experiencia del amor. La prudencia es la virtud base para el desarrollo de las demás virtudes morales, por ello, en el presente trabajo haremos una revisión de todos los aspectos que se deben considerar para el logro del desarrollo de esta virtud y procuraremos explicar cómo en la dinámica propia de cada familia se pueden llevar a cabo una serie de acciones que apunten a este objetivo.

Palabras clave: Familia, educación, virtud, prudencia.

\begin{abstract}
The achievement of virtue is perhaps one of the most difficult tasks to accomplish and yet is the purpose of education of the human person, the thing you should aim to reach its completion. This improvement to the human person is called, it can not achieve just using their own means, but requires the guidance and leadership of others who have been down the road for the development of virtue; the family is the place par excellence
\end{abstract}


where the human being can find this guide and driver of which we speak, is where the experience and development of virtues can be given in a natural way because this is the area where naturally gives the experience of love. Prudence is the virtue base for the development of the other moral virtues, therefore, in this paper we will review all aspects to be considered for achieving development of this virtue and seek to explain how in the dynamics of each family can carry out a series of actions aimed at this goal.

Key words: Family, education, virtue, prudence.

\section{Introducción}

La educación, según Santo Tomás, es la conducción de la prole hasta el estado perfecto del hombre en cuanto hombre, que es el estado de virtud (Martínez, 2002), en este sentido, queda claro que los padres son los primeros llamados a procurar la educación de sus hijos y que esta educación debe consistir, en primer lugar, en el logro de la virtud.

La prudencia es una virtud propia del entendimiento y a la vez una virtud que perfecciona la acción práctica de la persona, por lo tanto, es virtud intelectual y virtud moral; el ejercicio de la misma implica una rectitud en el obrar voluntario del hombre (Echavarría, 2005). Esta virtud es considerada madre de las demás virtudes morales, puesto que, para que la persona logre la ordenación de sus apetitos, requiere, en primer lugar, de una rectitud en el entendimiento que le permita tener una aproximación hacia la realidad basada en la verdad de las cosas y, además, necesita de cierta capacidad para obrar rectamente de acuerdo a cada circunstancia particular (Royo, 2012).

Es por esta razón que consideramos necesario profundizar en las bases teóricas de esta virtud, para, a partir de ello, procurar una comprensión de aquellas acciones concretas que los padres deben lograr que se pongan de manifiesto en la dinámica cotidiana de la familia para el desarrollo de la virtud en sus hijos.

La prudencia está conformada por partes cuasi integrales, que son: memoria, inteligencia, docilidad, sagacidad, razón, previsión, circunspección y precaución. En el presente trabajo haremos una explicación de cada una de ellas desde una aproximación basada en el pensamiento de Santo Tomás y procuraremos plantear situaciones concretas y ejemplos que nos den luces acerca de cómo los padres pueden, dentro de las vivencias cotidianas de la familia, desarrollar en sus hijos esta virtud.

Partimos de la premisa de que para poder educar a los hijos en virtudes es primordial la vivencia de las mismas por parte de los padres, es decir, ser un auténtico testimonio de persona virtuosa (Allers, 1950) y, en ese sentido, es necesario que los padres tengan un conocimiento verdadero acerca de las virtudes y sobre todo que comprendan que la posesión de las mismas no es algo a lo que sólo algunas personas están llamadas, 
sino una realidad que es posible de experimentar por todos los seres humanos, pero que requiere de un esfuerzo y compromiso.

Consideramos que este esfuerzo y compromiso se dará sólo cuando los padres comprendan la gran importancia que tiene en el perfeccionamiento de la persona el desarrollo de las virtudes morales y además entiendan cómo, de manera específica, mediante ciertas acciones concretas, se pueden desarrollar. El presente trabajo pretende servir a los padres para el logro de estos objetivos, especialmente para la comprensión de la virtud de la prudencia.

Este trabajo busca profundizar cómo puede llevarse a cabo la educación de la virtud de la prudencia en el seno familiar; para ello, se tomó como punto de referencia central el pensamiento de Santo Tomás de Aquino, sobre todo en lo referido al tratado de las virtudes morales, y se consideraron algunos autores contemporáneos que han estudiado la virtud de la prudencia a partir de la concepción clásica sobre la misma.

\section{Virtud de la prudencia}

La prudencia consiste en «cierta rectitud de discreción en toda clase de actos y de materias» (Tomás de Aquino, 2001, I-II, q. 61, a. 4), esta virtud es la 'madre' de las virtudes morales (Pieper, 2007, p.33); por lo tanto, para poder ser una persona virtuosa, el punto de partida ha de ser el desarrollo de esta primera virtud que «pertenece a la 'facultad cognoscitiva' (...) y radica propiamente en el entendimiento» (Tomás de Aquino, 2001, II-II, q. 47, a. 1), puesto que permite que la persona sea capaz de ver y entender la verdad de las cosas y su querer y su obrar estén de acuerdo a ella.

Sin embargo, la persona humana, en los inicios de su vida, es decir, en su infancia, no podrá aún internalizar esta virtud si no es capaz de ejercer un gobierno sobre sus apetitos; por ello es que la templanza y la fortaleza son aquellas virtudes que los padres deben hacer un gran esfuerzo por lograr en sus hijos en sus primeros años. Pero esto no quiere decir que se deba dejar la educación de la prudencia para cuando la persona ya tiene un gobierno de sus pasiones establecido, sino que esta debe darse a la par, ya que es la prudencia la que «perfecciona la rectitud impulsiva e instintiva del obrar» (Pieper, 2007, p. 37), es así que para que las demás virtudes se desarrollen se requiere primero de la acción de la prudencia. Podemos decir que nos encontramos frente a un círculo, en donde cada virtud ayuda a la otra a desarrollarse; pero, al ser la prudencia la virtud propia del entendimiento (Tomás de Aquino, 2001, II-II, q.47, a.1), es esta la que, a fin de cuentas, es la medida de las demás y es la que le suministra a «cada una el complemento que le permite el logro de su propia esencia; y todas participan de ella, alcanzando, a merced a tal participación, el rango de virtud» (Pieper, 2007, p. 39). Este círculo del que hablamos encuentra su solución en la prudencia de los padres; es la prudencia paterna la que dirige la educación de la fortaleza y templanza. El niño 
ciertamente no está en la capacidad de adquirir la prudencia en sus primeros años, es así que lo prudente en él es ser dócil a la prudencia de sus padres.

Para que la persona pueda obrar el bien es necesario que primero pueda conocer la realidad, por ello la virtud de la prudencia es esencial para el desarrollo de las demás virtudes porque es aquella que permite que la persona tenga una visión objetiva de la realidad y, a partir de esta, guiar su accionar. Para la ejecución del bien se requiere de la prudencia, pero, para que esta se desarrolle, la persona debe amar y querer el bien (Pieper, 2007, p. 75).

Ahora bien, la prudencia es una virtud que se va adquiriendo con la experiencia y para ello se requiere tiempo, por esta razón es tan importante el accionar de los padres, quienes van mostrando al hijo con sus propias acciones prudentes y con la manifestación del inmenso amor que sienten por ellos la verdad de las cosas, el valor de las mismas, y sobre todo los medios más adecuados para poder alcanzar el fin propuesto en cada situación concreta. Todas aquellas experiencias que el niño vaya teniendo a lo largo de su vida conformarán una serie de recuerdos que serán muy provechosos en el futuro para la toma de decisiones prudentes.

Las buenas acciones por sí mismas, sin una razón de ser, no llevan a la virtud; sólo aquellas acciones buenas que la persona realiza, pero sabiendo por qué las hace, para qué finalidad y bajo qué criterio de verdad, llevan a la virtud. En este sentido, los padres deben orientar a sus hijos sobre la razón de ser de las acciones que deben llevar a cabo, ya que no basta la acción buena, sino que la persona debe realizarla voluntariamente y - para que la voluntad tienda a un bien- el entendimiento debe primero mostrarle ese bien como tal.

\section{Formación de la virtud de la prudencia}

La formación de la virtud de la prudencia en el seno familiar no es tarea sencilla, «la vida familiar exige una actividad continuada. Esta actividad dificulta el proceso de reflexión y, como consecuencia, existe una tendencia a reaccionar frente a las situaciones nuevas que van surgiendo, más que a afrontarlas con serenidad para tomar decisiones acertadas» (Isaacs, 1996, p. 337); es en este contexto que en la vida cotidiana de la familia se van tomando una serie de decisiones específicas y, si los padres poseen la virtud de la prudencia, dichas decisiones tendrán congruencia con los valores que se quieren vivir en la familia; de lo contrario, puede ser que las decisiones tomadas por los padres sean fruto de la precipitación y no lleven a ningún fin coherente con la formación que desean brindar a sus hijos.

Para educar la prudencia es necesario que los padres desarrollen en sus hijos lo que Santo Tomás denomina partes cuasi integrales (Tomás de Aquino, 2001, II-II, q. 49) de 
la misma, entre ellas tenemos: la memoria, la inteligencia, la docilidad, la sagacidad, la razón, la previsión, la circunspección y la precaución.

La memoria ayuda al desarrollo de la prudencia, puesto que permite que la persona guarde y atesore todas aquellas experiencias que en algún momento de su vida la llevaron a una acción prudente. Los padres deben esforzarse por propiciar que su hijo pueda tener una gran cantidad de experiencias, porque para «conocer la verdad entre muchos factores, es necesario recurrir a la experiencia» (Tomás de Aquino, 2001, II-II, q. 49, a. 1) y esta se va formando del conjunto de recuerdos que la persona posee. Es por ello que, si los recuerdos de la persona sólo contienen vivencias negativas, imprudentes, acciones sin un sentido y finalidad, la persona no tendrá la posibilidad de ir forjando la virtud de la prudencia; en cambio, si en la vivencia cotidiana de la familia se experimentan muchas situaciones positivas, con una finalidad de orden moral, la persona tendrá en su haber una serie de recuerdos que le permitirán en un futuro tomar mejores decisiones y realizar una acción prudente. Este aspecto puede ser formado en la vivencia cotidiana de la dinámica familiar cuando los niños presencian y son observadores de las decisiones que toman sus padres y de las consecuencias que las mismas tienen según el contexto en que se dan. Al niño le llamará más la atención ciertas circunstancias y quedarán grabadas en su memoria; es así que los padres deben procurar que sean interesantes para sus hijos aquellas circunstancias que sean en sí mismas buenas y lleven a la vivencia de experiencias positivas, por ejemplo, las situaciones de juego serán buenos momentos a aprovechar para que el niño los recuerde en un futuro, y aquí podemos dar muchos ejemplos: las decisiones prudentes que pueden tomar los padres en ciertos juegos de mesa, las decisiones prudentes que toman los padres en juegos de representación de roles, las decisiones prudentes que toman los padres en la práctica de un deporte, etc., todas estas situaciones son formativas para el niño (Roqueñi, 20o8).

Por otro lado, algo que llama la atención del niño son los cambios de actividad y escenarios (visitas y paseos a lugares nuevos para él), en donde estén involucradas personas distintas a las que el niño está acostumbrado a ver cotidianamente (podría ser un primo mayor que es un modelo para el niño, o una persona con ciertas características personales peculiares), etc. En este sentido, los padres pueden aprovechar estas circunstancias peculiares para, con su ejemplo, ser un punto de referencia para el niño en la toma de decisiones prudentes, puesto que, en este contexto novedoso y atrayente para él, las vivencias quedarán más grabadas en su memoria. Así mismo, como ya dijimos anteriormente, en el día a día de la dinámica familiar, los padres deben ser un ejemplo constante de acciones prudentes y, si no lo fueran, los padres deben tener la capacidad de reconocer sus errores (lo más pronto posible) y, fomentar un diálogo con sus hijos en el que sean capaces de explicarles el porqué de su error y cuál es la acción que debió haber sido la más correcta, de esta forma quedará grabado 
en la memoria del niño el hecho de que, si alguien se equivoca, tiene opción, fruto de una reflexión, de enmendar su error.

La inteligencia es también parte integral de la prudencia, entendida como «inteligencia de los primeros principios» (Tomás de Aquino, 2001, II-II, q. 49, a. 2), es así que, para obrar rectamente, es necesario que la persona pueda tener un conocimiento de los primeros principios. De esta forma, los padres son los que deben procurar tener una claridad sobre los mismos en cada situación concreta que busquen dar a conocer a su hijo para, de esta manera, ir forjando en él el interés por llegar al conocimiento profundo de la verdad. La persona, para ser prudente, requiere de motivos, y el motivo central para poder actuar de manera prudente es el deseo de que las decisiones y acciones consecuentes estén acordes con la finalidad que se quiere alcanzar. «Se puede enfocar la virtud hacia el logro de la concordia social o hacia la eficacia en el trabajo, más si uno es cristiano, su motivo fundamental debe ser el cumplimiento de la Voluntad de Dios» (Isaacs, 1996, p. 339). Tomando en consideración esta finalidad de la que hablamos, es que los padres deben procurar tener un conocimiento profundo y real de aquella realidad que quieren alcanzar, para ello, deberán informarse con las fuentes más válidas, deberán cultivar la virtud de la estudiosidad y lo mismo deberán procurar que hagan sus hijos, partiendo siempre de la premisa de que la verdad es una (no depende de la opinión o criterio de cada quien), y toda la realidad se ordena a la Verdad. Un hábito que desde pequeños se debe procurar desarrollar en los niños es el de la lectura, para ello, será necesario que en casa sea normal la presencia de libros, revistas, etc., de temáticas distintas para cada edad, también deberá ser algo cotidiano que los hijos vean a sus padres leyendo y comentando lo que leen, por ejemplo, una noticia en el periódico puede ser motivo de reflexión, de crítica y diálogo entre los miembros de la familia; estas situaciones van cultivando el desarrollo intelectual del niño, ya que le ayudan a discernir, a tener criterios, a enjuiciar la realidad y, por lo tanto, a tomar decisiones basadas en un análisis profundo de la misma, la cual le fue mostrada por sus padres de manera natural y sencilla en cada momento y situación cotidiana familiar.

La docilidad es otra de las partes de la prudencia que se forja esencialmente en el seno familiar, esta consiste en que la persona esté bien dispuesta para «recibir la instrucción de otros» (Tomás de Aquino, 2001, II-II, q. 49, a. 3), es decir, tomar en cuenta el consejo y orientación que otros puedan hacerle, puesto que ya han vivido más experiencias a lo largo de su vida y por ello son más prudentes. La persona dócil es aquella que sabe escuchar a los demás y, a su vez, meditar sobre las recomendaciones que los otros le hacen para, a partir de esto, tomar sus propias decisiones. Los padres que aconsejen y orienten al hijo con amor, comprensión y sin imposiciones, buscando más bien el diálogo y procurando explicar el porqué de sus recomendaciones, podrán con más facilidad lograr la docilidad necesaria en sus hijos, la que debe ser «desarrollada y perfeccionada con mucho esfuerzo» (Palet, 2007a, p. 81), ya que la persona muchas 
veces tiene una tendencia (fruto del pecado original) a la soberbia y rebeldía, lo que la lleva a no permitir que «otros le digan lo que debe hacer».

Los niños pequeños, por el mismo hecho de serlo, han vivido muy pocas experiencias y tienen un escaso conocimiento de la realidad, estos son algunos de los factores que les incapacita para tomar decisiones; es así que deben seguir la guía de sus padres, sobre todo en aquellas situaciones que en sí mismas sean complejas para ellos, ya sea porque no cuentan con la información adecuada o no pueden poseerla por su corta edad o porque se trata de una situación nueva que no han tenido oportunidad de vivir anteriormente (Isaacs, 1996); es por ello que, para que los niños sean dóciles con los padres, estos deben ser un punto de referencia para ellos, esto es, deben contar con la información adecuada para cada situación y tomar decisiones acertadas basadas en la realidad de las cosas, resolviendo los problemas concretos que se les presenten, de tal forma que los niños puedan confiar en que sus padres realmente los van a ayudar a resolver las dificultades que puedan tener, porque ven que ellos son capaces de resolver sus propias dificultades, es decir, son un ejemplo para ellos. La docilidad tiene como punto de partida esto: la confianza que el niño tiene en que sus padres «saben» qué hacer en determinadas situaciones; si el niño no confía en las capacidades de sus padres, difícilmente les obedecerá y mucho menos les pedirá consejo cuando él no tenga claro cuál debe ser su proceder.

Por otro lado, otro aspecto necesario para desarrollar esta confianza que el niño debe tener hacia sus padres, además de lo ya visto (la no imposición de las normas y la seguridad de que los padres saben resolver los problemas), está el hecho de que el niño debe tener la total certeza de que las orientaciones y consejos de sus padres buscan su bien, aquí nuevamente la confianza es la base, puesto que, si el niño no confía en que sus padres quieren su bien, probablemente no seguirá sus indicaciones; por ello es que es muy importante que los padres tengan una recta intención en cada indicación y consejo que dan a sus hijos, por ejemplo, si una madre le dice a su hijo «pórtate bien, porque ahí está mi jefe y no quiero que piense que eres un malcriado», es lógico que el niño percibirá inmediatamente que la intención de su madre es quedar bien ante su jefe y probablemente no le obedezca, porque no ve que su madre tenga la intención de ir forjando en él un buen comportamiento que, a la larga, será bueno para él, porque lo ayudará a ser mejor persona y a saber comportarse adecuadamente en una situación social.

Como vemos, la confianza en sus padres es un punto esencial en el desarrollo de la docilidad, la cual es sumamente importante, puesto que, si el niño desde pequeño la desarrolla, cuando sea adolescente, le será más fácil escuchar el consejo de sus padres, maestros o de otras personas que puedan realmente guiarlo por un buen camino y esto será de gran ayuda para la toma de decisiones prudentes a lo largo de su vida. 
La sagacidad «es un hábito por el que de pronto se sabe hallar lo que conviene» (Tomás de Aquino, 2001, II-II, q.49, a.4); en algunas ocasiones la persona se ve en la necesidad de actuar respondiendo de manera inmediata, debido a que, si no actúa en el momento, puede perjudicar a otros o a sí mismo; es en estas circunstancias que la virtud de la sagacidad se pone de manifiesto, ya que, por medio de la misma, la persona deberá actuar prudentemente, pero no tendrá el tiempo suficiente para reflexionar sobre la situación concreta a la que tiene que hacer frente. En este sentido se hace necesario que la persona tenga una base sólida sobre principios universales que le permita estar en posesión de información adecuada para cada situación en particular; además es importante que la persona se encuentre ejercitada en la toma de decisiones rápidas e inmediatas. Para educar esta virtud de la sagacidad es importante (como en la educación de todas las virtudes) el ejemplo que los padres brinden a sus hijos a partir de su propio accionar, tomando decisiones correctas que permitan la resolución de conflictos de manera inmediata; así mismo, es importante que los padres permitan que sus hijos tomen sus propias decisiones, pero siempre a partir del consejo y recomendaciones oportunas que ellos les puedan brindar, por otro lado, no deben descuidar el hecho de ir forjando en sus hijos una visión del mundo realista, de tal forma que ellos puedan aproximarse a la realidad con una mirada global e integral de la misma. Si los padres sobreprotegen a sus hijos, no podrán ayudarlos a desarrollar este hábito necesario para la acción prudente, por ello es recomendable que en el seno familiar se permita a los hijos desenvolverse con iniciativa y que puedan desarrollar su creatividad para resolver sus problemas.

La razón, como es lógico, es parte integrante de la prudencia, puesto que, para la acción prudente, la persona primero tiene que haber hecho un razonamiento sobre la situación a considerar, para luego obrar. Considero que la acción de la familia debe ir en la misma línea de la sagacidad, es decir evitar la sobreprotección para con los niños y más bien permitir situaciones en las que ellos deban buscar las distintas posibilidades de solución a las diversas circunstancias que les toca afrontar; sin embargo, a diferencia de la forja de la sagacidad, en la forja de la razón, los padres deben procurar instancias en las que sus hijos puedan dedicar un tiempo en la preparación de ciertos temas que les permitan aproximarse a la realidad de una manera profunda.

En este aspecto, los padres deben ayudar a sus hijos a desarrollar las capacidades necesarias para, en primer lugar, recoger la información adecuada para cada situación; para ello será necesario que ejerciten su capacidad de observación, su capacidad de escucha y su capacidad de comprensión de lo leído. Un siguiente paso será ayudarlo a distinguir entre hechos y opiniones, entre lo importante y lo secundario, ayudarlo a reconocer sus propios prejuicios, a analizar críticamente la información recibida, a reconocer qué información es necesaria en cada caso, etc.; una buena forma de lograr esto puede ser mediante el diálogo de películas, programas de televisión, obras de 
teatro, noticieros, obras literarias, así como situaciones cotidianas que puedan invitar a la reflexión (Isaacs, 1996).

La previsión «es la parte principal de la prudencia, ya que todas las demás cosas requeridas para ella son necesarias a efectos de ordenar algo directamente al fin» (Tomás de Aquino, 2001, II-II, q. 49, a. 6); de esta manera, la persona previsora es aquella que ordena los medios de tal forma que tengan una jerarquización de acuerdo a la finalidad buscada, por lo tanto, su accionar será prudente en la línea de no desperdiciar las oportunidades ni circunstancias que se le presenten en acciones desordenadas o sin dirección alguna. Los padres son los primeros que con su ejemplo deben enseñar a sus hijos la previsión; si ellos actúan ordenadamente, siguiendo un criterio lógico y escogiendo los medios más adecuados para alcanzar sus metas, les mostrarán a sus hijos este orden que deben seguir para poder alcanzar la rectitud en su obrar. En este sentido, ciertas costumbres de la familia, que de alguna manera marcan la pauta "rutinaria" de la misma, son importantes, debido a que seguir cierta rutina en el día a día ayuda al niño a tener la noción de la importancia de prepararse para las situaciones que están por venir. Es importante que en casa el niño viva la experiencia de seguridad que brinda el hecho de cumplir con ciertos horarios y saber qué es lo que toca hacer en determinados momentos del día.

Los padres también educan esta virtud en sus hijos cuando en casa procuran el cumplimiento de actividades para las cuales debe haber una preparación, por ejemplo, cuando hay invitados en casa, existe una preparación para recibir adecuadamente a los mismos, cuando la familia va a viajar, existe una preparación de todo lo necesario para el viaje y así evitar imprevistos de último momento, etc.

La circunspección es la que permite que la persona «compare lo que se ordena al fin con las circunstancias» (Tomás de Aquino, 2001, II-II, q. 49, a. 7), vale decir, actúe de acuerdo a las circunstancias, es así que en determinadas situaciones uno deberá evitar ciertas actitudes y comportamientos que probablemente sí sean pertinentes en otras situaciones; esta característica es propia de la persona prudente. Para que el niño desarrolle la circunspección se hace necesario que actualice otras partes de la prudencia como la docilidad y la razón, entre otras, ya que de esta manera será capaz de dejarse guiar por el consejo de los demás y así podrá darse cuenta con mayor facilidad de cada circunstancia concreta que le toque vivir y de cuál debe ser el comportamiento más adecuado para ella. En esta línea, la explicación que dan los padres a sus hijos acerca de su propio comportamiento y del por qué, en determinada situación, actuaron de una manera distinta a otra situación es de gran significado para el niño, quien podrá darse cuenta de que la decisión de comportarse de determinada forma no es invariable, sino que se hace necesario considerar ciertos criterios para evaluar cada situación en particular. Los padres deben ir dando criterio a los hijos, de tal forma que estos sepan qué criterio deben usar en cada momento, por ejemplo, 
criterio para el comportamiento en casa, criterio para enjuiciar actos de los demás, criterio para enjuiciar si es conveniente o no hacer o dejar de hacer algo, criterio para enjuiciar problemas sociales y personales, criterio para saber si se está actuando con justicia, sinceridad, respeto, etc., «en síntesis, se tratará de ayudar a los hijos a enjuiciar según las reglas del juego establecidas, según las leyes comunes y ordinarias y según principios más elevados» (Isaacs, 1996, pp. 439-350).

La precaución se hace necesaria para poder ser prudente, porque en las diversas situaciones que a la persona le toca afrontar no siempre está presente sólo el bien, sino que muchas veces el mal lo está y, por ello, la persona debe actuar de manera precavida, para poder evitar el mal cuando sea pertinente. En las vivencias cotidianas de la familia, desde que el niño es muy pequeño se le inculca la precaución, sobre todo en lo relativo al cuidado corporal, por ejemplo cuando los padres le dicen al niño: «no vayas por ahí porque te puedes caer»y otras indicaciones parecidas. Este tipo de recomendaciones es de vital importancia para que el niño pueda hacer una valoración adecuada de la realidad en cada una de las circunstancias que le toca afrontar, pero se hace necesaria cierta moderación, porque, de lo contrario, lo que se estará logrando es un niño temeroso; por eso las recomendaciones y consejos de los padres siempre deben ser acordes con la razón y verdad de las cosas.

Como podemos ver, si bien para poseer la prudencia se requiere de experiencia y cierta madurez, desde los primeros años de vida debe forjarse el desarrollo de la misma y esta labor corresponde que se realice en el seno de la familia, ya que son los padres quienes deben poner los medios necesarios para que el niño aprenda a comprender la verdad de las cosas y a actuar acorde a ella. Pilar fundamental para ello es que los padres sean personas prudentes para que puedan juzgar adecuadamente las situaciones que requieren de su consejo y guía que permitan en el niño «progresar en el proceso de adquisición de los hábitos deliberativos que acompañan a todo acto prudente» (Palet, 2007b, p. 83).

Fomentar en los hijos la virtud de la prudencia implica todo un proceso de enseñanza en la toma de decisiones acertadas; los niños, desde pequeños, en las situaciones que viven en su familia, con sus amigos, en su escuela, van tomando decisiones que implican una reflexión acerca de su actuar. Los padres:

ayudan a los hijos: primero, procurando que éstos comprendan y asuman personalmente sus órdenes; luego, ayudándoles a considerar distintas alternativas y, por fin, preguntándoles para asegurarse de que los hijos consideran seriamente las opciones antes de decidir ellos mismos. Aquí no hay recetas. El riesgo de dejar a los hijos decidir por su cuenta tiene que ser calculado. (Isaacs, 1996, p. 352) 
Los padres podrán darse cuenta de que su hijo está aprendiendo a ser prudente si es que les pide consejo sobre algunas situaciones de su vida, si busca fuentes adecuadas, si reflexiona sobre la información recibida, si dialoga sobre sus criterios para poder medir las consecuencias de sus actos, etc., es decir, si toma en cuenta muchos aspectos de la situación antes de decidir y actuar conforme a esta decisión.

\section{Conclusiones}

La prudencia es una virtud propia del entendimiento y, además, una virtud moral, porque para su pleno ejercicio se requiere de una comprensión profunda y veraz acerca de la realidad y, a partir de ello, la posibilidad de una toma de decisiones que den lugar a un recto obrar en la persona.

La prudencia está conformada por partes cuasi integrales de la misma, las cuales son necesarias desarrollar para poder ser personas prudentes. Estas partes cuasi integrales de la prudencia son: memoria, inteligencia, docilidad, sagacidad, razón, previsión, circunspección y precaución.

La prudencia es una virtud que, para que la persona pueda poseerla, es necesario que pase por una acción educativa; el lugar por excelencia para que se dé esta acción educativa es la familia.

\section{Referencias}

Allers, R. (1950). Naturaleza y educación del carácter. Barcelona, España: Editorial Labor.

Aquino, Tomás de. (2001). Suma de teología. Madrid, España: Biblioteca de Autores Cristianos (BAC).

Echavarría, M. (2005). La praxis de la psicología y sus niveles epistemológicos según Santo Tomás de Aquino. Barcelona, España: Documenta Universitaria.

Isaacs, D. (1996). La educación de las virtudes humanas. Navarra, España: EUNSA.

Martínez, E. (2002). Persona y educación en Santo Tomás de Aquino. Barcelona, España: Fundación Universitaria Española.

Palet, M. (2007a). La educación de las virtudes en la familia. Barcelona, España: Ediciones Scire.

Palet, M. (2007b). La familia educadora del ser humano. Barcelona, España: Ediciones Scire.

Pieper, J. (2007). Las virtudes fundamentales. Madrid, España: Ediciones Rialp.

Roqueñi, J. (2008). Educación de la afectividad. Una propuesta desde el pensamiento de Tomás de Aquino. Navarra, España: EUNSA.

Royo, A. (2012). Teología de la perfección cristiana. Madrid, España: Biblioteca de Autores Cristianos (BAC). 